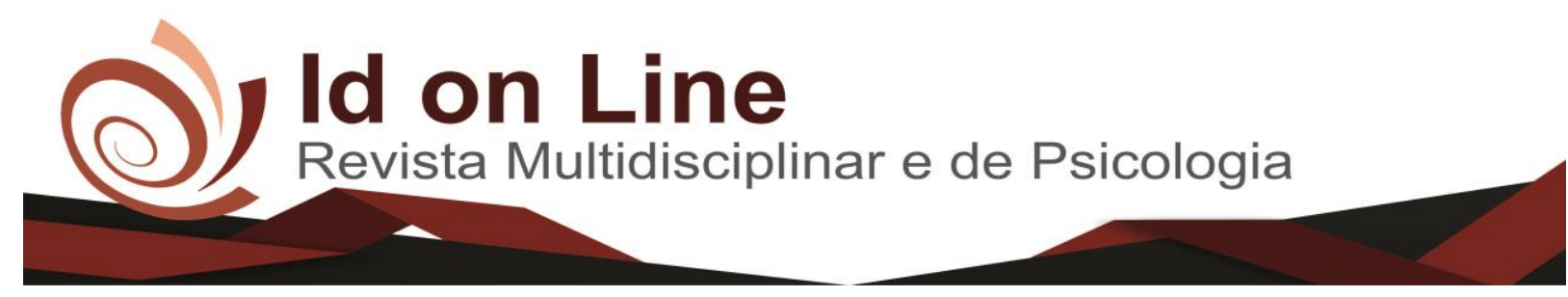

Artigo de Revisão

\title{
Sistematização da Assistência de Enfermagem em Mulheres com Sintomatologia Depressiva: Uma Revisão Sistemática
}

Thainá Emi Barreto Gomes ${ }^{1}$; Olguimar Pereira Ivo ${ }^{2}$

Resumo: A depressão, mais conhecida como o mal do século, é uma doença de caráter público que tem crescido no decorrer dos anos. Metodologia: Trata-se de uma revisão sistemática da literatura baseada em evidências científicas, elencadas por meio de publicações, com informações que validam originalmente os resultados de pesquisas qualitativas ou quantitativas. Os bancos de dados utilizados foram: Biblioteca Virtual de Saúde (BVS), LILACS e Google Acadêmico. Resultados: as assistências de enfermagem aplicadas em mulheres com depressão encontradas nesta revisão encontradas foram: escuta qualificada, musicoterapia, terapia cognitiva comportamental, biodança e auxilio na terapia medicamentoso. Conclusão: Entende-se que os resultados dos estudos analisados mostram a importância do profissional da enfermagem no cuidado e acolhimento dessas pacientes.

Palavras-chave: Depressão; Mulheres; Sistematização da assistência de enfermagem.

\section{Systematization of Nursing Assistance in Women with Depressive Symptomology: A Systematic Review}

\begin{abstract}
Depression, better known as the evil of the century, is a disease of a public character that has grown over the years. Methodology: This is a systematic review of the literature based on scientific evidence, published through publications, with information that originally validates the results of qualitative or quantitative research. The databases used were: Virtual Health Library (VHL), LILACS and Google Scholar. Results: the nursing assistances applied in women with depression found in this review were: qualified listening, music therapy, cognitive behavioral therapy, biodance and help in drug therapy. Conclusion: it can be to understood that the results of the studies analyzed show the importance of the nursing professional in the care and hospitalization of these patients.
\end{abstract}

Keywords: Depression; Women; systematization of nursing care.

\section{Introdução}

A depressão, mais conhecida como o mal do século, é uma doença de caráter público que tem crescido no decorrer dos anos, atingindo boa parte da população mundial. Os números são alarmantes, e suas consequências podem levar à invalidez laboral e até ao suicídio.

\footnotetext{
${ }^{1}$ Graduanda em Enfermagem pela Faculdade Independente do Nordeste (FAINOR). Vitória da Conquista, Bahia, Brasil. E-mail: thaina.emi@gmail.com

${ }^{2}$ Graduada em enfermagem pela Universidade Estadual do Sudoeste da Bahia, Mestre em Ética e Gestão, docente dos cursos de Enfermagem e Fisioterapia da Faculdade Independente do Nordeste. Vitória da Conquista, Bahia, Brasil E-mail: olgaivo13@ gmail.com

Autor correspondente:

Thainá Emí Barreto Gomes. Av. Luis Eduardo Magalhães, 1305 - Candeias, Vitória da Conquista - BA, (77) 3161-1000,
} 
Segundo Pereira et al. (2017), por seu alto nível de incidência em todo o mundo, a depressão é um dos fenômenos do século que mais tem chamado atenção, também por se tratar de uma questão de saúde pública.

De acordo com a Classificação de Transtornos Mentais e de Comportamento da CID10 (OMS, 1993), em episódios depressivos típicos, incluindo as variedades descritas como leve, moderada e grave, o indivíduo usualmente sofre de humor deprimido, perda de interesse, diminuindo as atividades e reduzindo a energia, que leva a um aumento da fadiga, e, mesmo após esforços leves é observado um cansaço marcante.

Segundo Zammit e Owen (2006) as reações biológicas ao estresse é uma hipótese que explica a maior prevalência de depressão nas mulheres do que nos homens. Para Hofmann et al (2010), isso pode ser explicado pelas duplas jornadas de trabalho exercidas pelas mulheres. E, de acordo com Southwick e Charney (2012) essa diferença é explicada pelo modo de enfrentamento da doença que as mulheres se submetem. Situação em que os autores consideraram ineficaz.

Santiago e Holanda (2013) citam que algumas pesquisas indicam que fatores substanciais como histórico familiar, episódio anterior de depressão, doença física, perdas, acontecimentos estressantes, abuso de medicamentos ou drogas, podem aumentar o risco para a depressão.

Sobre o conceito de depressão, o DSM (2013) a define como uma doença médica que altera de forma negativa a maneira como a pessoa sente, como pensa e como age diante das situações. Sabe-se que a depressão é de origem interna por conta de alterações em neurotransmissores, como explica a American Psychiatric Association-APA(2005). Segundo a Associação Brasileira de Psiquiatria-ABP (2014), podem existir influências ambientais, como problemas financeiros e emocionais, que podem iniciar um quadro depressivo.

Essa situação alarmante acontece nas Unidades Básicas de Saúde (UBS) e Estratégias de Saúde da Família (ESF), pois são as portas de entrada para os serviços de saúde no país (GUEDES et al., 2015). Sendo assim, os profissionais devem levar em conta o ser humano como um ser singular, complexo, integral e que está inserido em grupos socioculturais, buscando promover a saúde, prevenir e tratar enfermidades, também reduzir danos ou sofrimentos que possam impedi-lo de ter um viver saudável. A partir dessa premissa, deve-se acolher todo e qualquer cidadão, usuário assíduo ou não da unidade, em sua totalidade, de forma 
holística.

Segundo Waidman et al (2012), está incluso nas obrigações do enfermeiro prestar assistência à pessoas com transtornos mentais, mas também a desenvolver ações que auxiliam na prevenção e no diagnóstico precoce, de forma individual e coletiva, pessoa e família.

A ideia da realização do presente estudo surgiu a partir dos relatos dos Agentes Comunitários de Saúde (ACS) que atuam em uma Unidade de Saúde da Família (USF) de uma cidade do sudoeste da Bahia, pois percebeu-se que existe um alto índice de mulheres com sintomas depressivos e/ou que relatam o uso de medicações para tais sintomas na área que abrange a referida USF. Nesta perspectiva, o presente estudo tem por objetivo geral: averiguar a sistematização da assistência do enfermeiro em mulheres com sintomas depressivos, e por objetivos específicos: identificar na literatura as medidas implementadas pelo enfermeiro em Unidades Básicas de Saúde, para mulheres com sintomatologia depressiva; e identificar na literatura o impacto do atendimento às mulheres com sintomatologia depressiva em Unidades de Saúde.

\section{Metodologia}

Trata-se de uma revisão sistemática da literatura baseada em evidências científicas, elencadas por meio de publicações, com informações que validam originalmente os resultados de pesquisas qualitativas ou quantitativas.

Assim, a pesquisa qualitativa proporciona um aprofundamento da busca do porquê de algo, com o intuito de mostrar também o que deve ser feito em determinada situação (GERHARDT; SILVEIRA, 2009). Já a pesquisa quantitativa se diferencia da qualitativa, pois busca objetividade por meio da quantificação dos dados com a linguagem matemática, segundo Fonseca (2002).

Foram importantes os dois tipos de abordagens, quantitativa e qualitativa, pois proporcionaram maior abrangência na busca dos dados nos estudos científicos elencados. Neles foram coletados os dados para a pesquisa, com intuito de envolver aqueles considerados quantificáveis e explicativos.

Para nortear o tema escolhido, foi feita a pergunta norteadora: "Qual a assistência especifica da equipe de enfermagem nas mulheres que apresentam sintomas depressivos?”.

Após a elaboração da pergunta norteadora, iniciou-se a pesquisa e coleta dos artigos. A 
pesquisa foi realizada nos bancos de dados da Biblioteca Virtual de Saúde (BVS), onde utilizouse como descritor, depois da busca no DeCS (Descritores de Saúde), a palavra "depressão" e como palavras-chave "cuidados de enfermagem" e "mulheres". No banco de dados do LILACS e Google Acadêmico, as palavras-chave foram “depressão" e "cuidados de enfermagem”. Os achados da pesquisa (Tabela 1) foram analisados, conforme os critérios de inclusão e exclusão pré-determinados, e destrinchados para melhor compreensão de seus resultados, conforme apresentado nas figuras 1,2 e 3 .

Figura 1- Distribuição da metodologia de busca de artigos na base BVS.

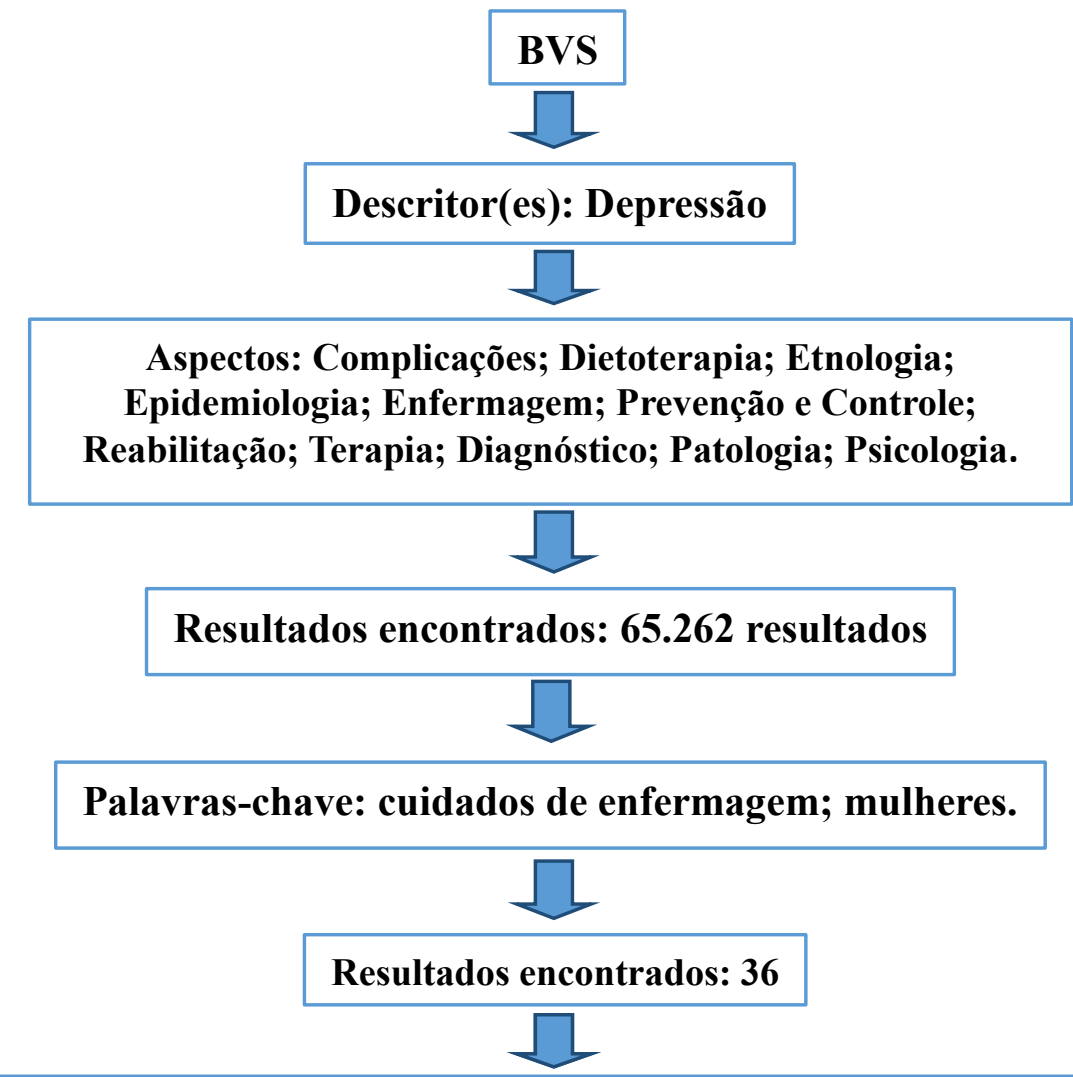

Filtros selecionados: MEDLINE; Depressão; Cuidadores; Mulheres; Saúde Mental; Saúde da Mulher; Ansiedade; Enfermagem Psiquiátrica; Atitude Frente à Saúde; Continuidade da Assistência ao paciente; Aconselhamento; Espiritualidade. Aspecto clínico: Prognóstico; Terapia. Limite: Humanos; Feminino. Assunto da revista: Enfermagem; Psicologia; Saúde da Mulher.

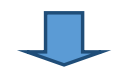

Resultados encontrados: 13

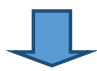

Artigos utilizados na pesquisa: 3 
Figura 2- Distribuição da metodologia de busca de artigos na base LILACS

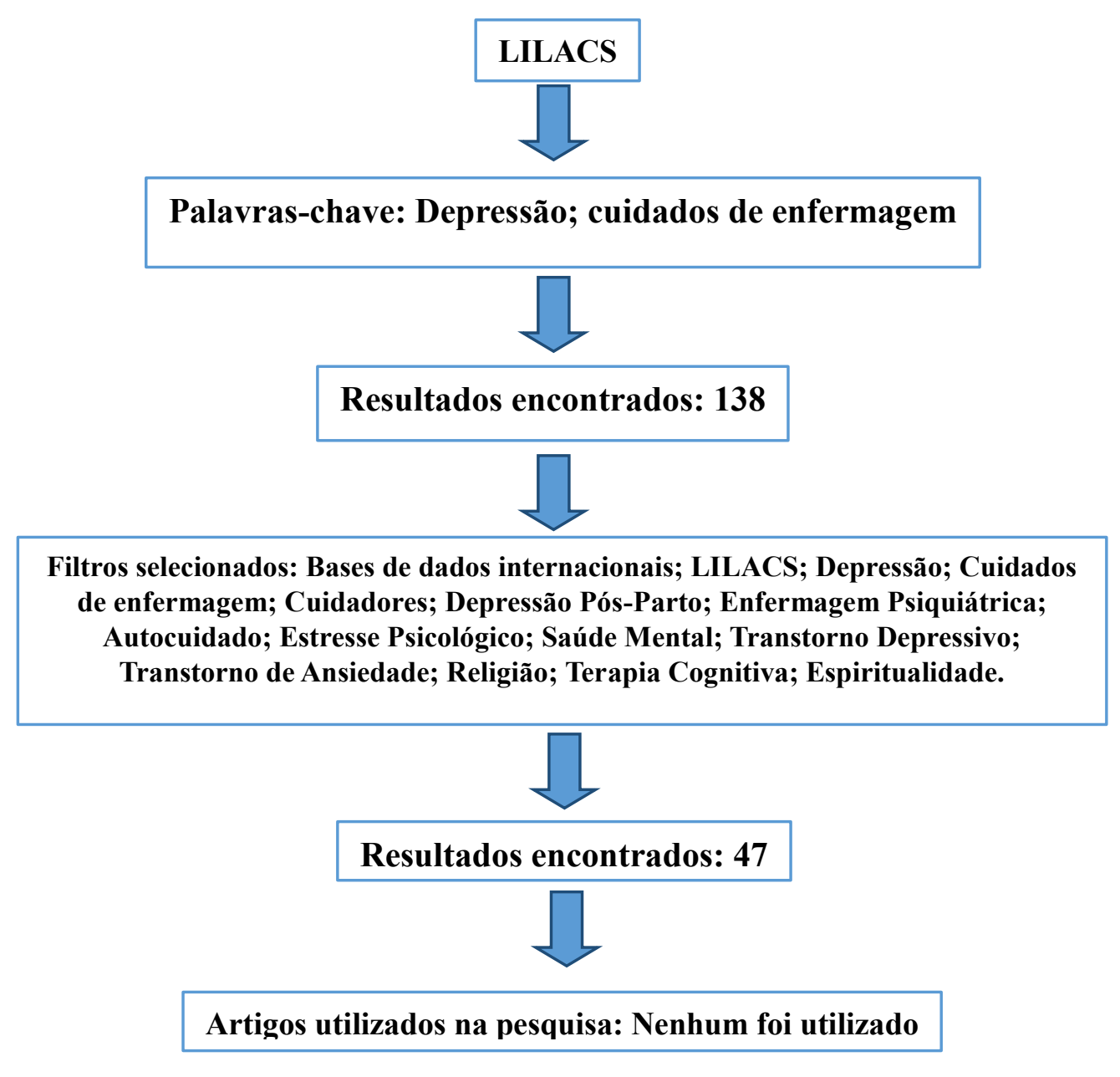

Figura 3- Distribuição da metodologia de busca de artigos na base Google Acadêmico

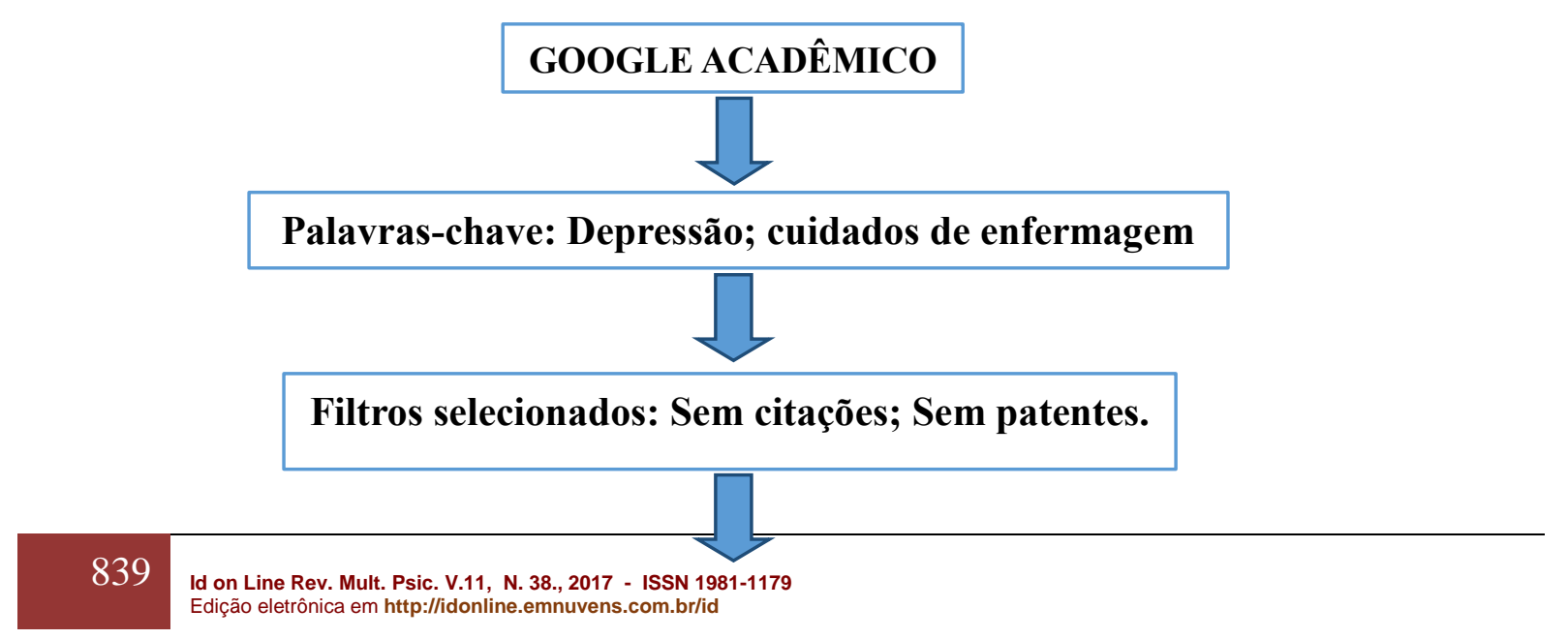




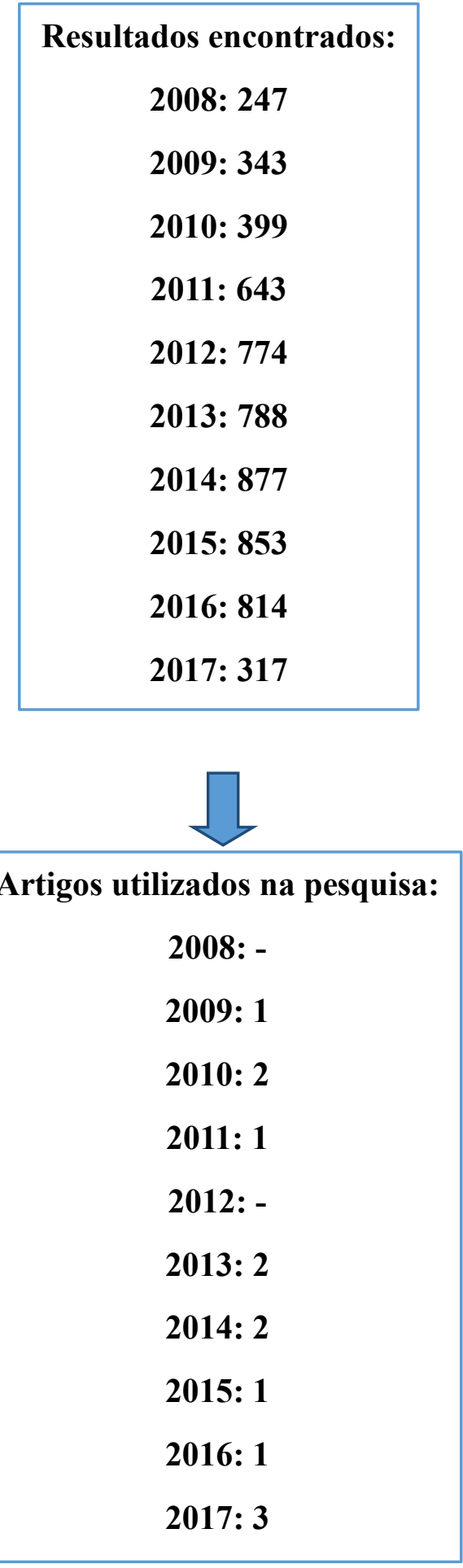




\section{Resultados e Discussão}

Foram encontrados na busca na BVS 13 artigos que, após avaliação e submissão dos critérios de inclusão e exclusão, foram utilizados na pesquisa, 3. Na base de dados do LILACS foram encontrados 47 artigos, porém nenhum foi utilizado para a pesquisa. No Google Acadêmico, no período de 2008 a 2017, foram encontrados 6.055 artigos e foram utilizados para a pesquisa, 13 artigos. Os artigos selecionados para a pesquisa se apresentam nas línguas inglês e português. Na Tabela 1 os achados foram destrinchados de acordo com a base de dados em que foram encontrados e o ano.

Tabela 1- Síntese dos artigos encontrados na revisão segundo periódico, título, autores, ano, abordagem, tipo de análise e base de dados.

\begin{tabular}{|c|c|c|c|}
\hline Periódico & Título & Autores / Ano & $\begin{array}{c}\text { Abordagem / } \\
\text { Tipo de análise / } \\
\text { Base de dados }\end{array}$ \\
\hline $\begin{array}{l}\text { The Nurse Practitioner. Vol. } \\
\text { 40, No. } 7\end{array}$ & $\begin{array}{l}\text { Prenatal depression: } \\
\text { Early intervention }\end{array}$ & $\begin{array}{l}\text { ANDERSON CA, } \\
\text { LIESER C. } \\
2015\end{array}$ & $\begin{array}{l}\text { Qualitativo/ } \\
\text { BVS }\end{array}$ \\
\hline $\begin{array}{l}\text { JournalofGerontologicalNursi } \\
\text { ng• Vol. 36, No. 9, } 2010\end{array}$ & $\begin{array}{c}\text { CollaborativeCareModels for Late-Life } \\
\text { DepressionandAnxiety }\end{array}$ & $\begin{array}{l}\text { SMITH M. } \\
2010\end{array}$ & BVS \\
\hline $\begin{array}{l}\text { InternationalJournalofNursing } \\
\text { Studies } 48 \text { (2011) 3-12 }\end{array}$ & $\begin{array}{c}\text { The effects of a tailored cardiac } \\
\text { rehabilitation program on depressive } \\
\text { symptoms in women: A randomized clinical } \\
\text { trial }\end{array}$ & $\begin{array}{l}\text { BECKIE TM, et } \\
\text { al. } \\
2011\end{array}$ & $\begin{array}{l}\text { Qualitativo/ } \\
\text { Ensaio Clínico } \\
\text { Randomizado/ } \\
\text { BVS }\end{array}$ \\
\hline $\begin{array}{c}\text { Dados Internacionais de } \\
\text { Catalogação-na-Publicação } \\
\text { (CIP) } \\
\text { (Biblioteca Frei Eugênio, } \\
\text { Universidade Federal do } \\
\text { Triângulo Mineiro, MG, } \\
\text { Brasil) }\end{array}$ & $\begin{array}{c}\text { Ações dos Enfermeiros em Saúde Mental } \\
\text { na } \\
\text { Estratégia Saúde da Família }\end{array}$ & $\begin{array}{l}\text { GONÇALVES } \\
\text { RMDA. } \\
2009\end{array}$ & $\begin{array}{l}\text { Qualitativo/ } \\
\text { Descritivo } \\
\text { transversal/ } \\
\text { Google } \\
\text { Acadêmico. }\end{array}$ \\
\hline $\begin{array}{l}\text { Revista de Pesquisa: Cuidado } \\
\text { é Fundamental Online }\end{array}$ & $\begin{array}{c}\text { O cuidado de enfermagem e sua } \\
\text { contribuição para prevenir a depressão pós- } \\
\text { parto na adolescência }\end{array}$ & $\begin{array}{l}\text { FONTES FS, et } \\
\text { al. } \\
2010\end{array}$ & $\begin{array}{l}\text { Qualitativa/ } \\
\text { Descritivo/ } \\
\text { Google } \\
\text { Acadêmico }\end{array}$ \\
\hline $\begin{array}{l}\text { Universidade Fernando } \\
\text { Pessoa; Faculdade de } \\
\text { Ciências da Saúde. }\end{array}$ & $\begin{array}{l}\text { A utilização da música como coadjuvante } \\
\text { terapêutico na Saúde Mental e Psiquiatria. }\end{array}$ & $\begin{array}{l}\text { CARDOSO AJS. } \\
2010\end{array}$ & $\begin{array}{l}\text { Quantitativo/ } \\
\text { Exploratório, } \\
\text { transversal/ } \\
\text { Google }\end{array}$ \\
\hline
\end{tabular}


Acadêmico

\begin{tabular}{|c|c|c|c|}
\hline $\begin{array}{l}\text { Universidade Católica } \\
\text { Portuguesa } \\
\text { Instituto de Ciências da Saúde }\end{array}$ & $\begin{array}{l}\text { Intervenção em Enfermagem de saúde } \\
\text { mental e Psiquiátrica num centro de dia do } \\
\text { concelho de Oeiras }\end{array}$ & Dias MMG. & $\begin{array}{l}\text { Quantitativo/ } \\
\text { Exploratório/ } \\
\text { Google } \\
\text { Acadêmico }\end{array}$ \\
\hline $\begin{array}{c}\text { Revista de Enfermagem } \\
\text { Referência. }\end{array}$ & $\begin{array}{l}\text { Análise compreensiva de uma intervenção } \\
\text { na ansiedade e depressão em doentes } \\
\text { hospitalizados com insuficiência cardíaca }\end{array}$ & $\begin{array}{c}\text { MENDES } \\
\text { AMOC; } \\
\text { EUFRÁSIO MLP. } \\
2013\end{array}$ & $\begin{array}{l}\text { Qualitativo/ } \\
\text { Estudo de caso/ } \\
\text { Google } \\
\text { Acadêmico }\end{array}$ \\
\hline $\begin{array}{l}\text { C\&D-Revista Eletrônica da } \\
\text { Fainor, Vitória da Conquista, } \\
\text { v.6, n.2, p.175-187, jul./dez. } \\
2013\end{array}$ & $\begin{array}{l}\text { Atuação do Enfermeiro do } \\
\text { Programa de saúde da Família frente ao } \\
\text { indivíduo portador de transtorno mental }\end{array}$ & $\begin{array}{l}\text { REIS LA; } \\
\text { BRITO FR; } \\
\text { MOREIRA VS; } \\
\text { AGUIAR ACSA } \\
2013\end{array}$ & $\begin{array}{c}\text { Qualitativo/ } \\
\text { Descritivo/ } \\
\text { Google } \\
\text { Acadêmico }\end{array}$ \\
\hline & $\begin{array}{c}\text { Editorial: Consensos em Saúde Mental - } \\
\text { Diagnósticos, } \\
\text { Intervenções e Resultados }\end{array}$ & $\begin{array}{l}\text { SEQUEIRA, C. } \\
2014\end{array}$ & \\
\hline
\end{tabular}

\begin{tabular}{|c|c|c|c|}
\hline $\begin{array}{c}\text { Escola Superior de } \\
\text { Enfermagem de Lisboa }\end{array}$ & $\begin{array}{l}\text { As Técnicas de Relaxamento como } \\
\text { Instrumento } \\
\text { Terapêutico de Enfermagem em Pessoas } \\
\text { com } \\
\text { Sofrimento Mental }\end{array}$ & Elias MTMNC. & $\begin{array}{l}\text { Qualitativo/ } \\
\text { Intervenção/ } \\
\text { Google } \\
\text { Acadêmico }\end{array}$ \\
\hline $\begin{array}{l}\text { Instituto Politécnico de } \\
\text { Leiria- Escola Superior de } \\
\text { Saúde }\end{array}$ & $\begin{array}{l}\text { REDESCOBRINDO A ENFERMAGEM } \\
\text { DE SAÚDE MENTAL }\end{array}$ & $\begin{array}{l}\text { DE CARVALHO } \\
\text { DRS. } \\
2015\end{array}$ & $\begin{array}{l}\text { Qualitativo/ } \\
\text { Descritivo, } \\
\text { crítico-reflexivo/ } \\
\text { Google } \\
\text { Acadêmico }\end{array}$ \\
\hline $\begin{array}{l}\text { UNIVERSIDADE DE } \\
\text { ÉVORA } \\
\text { ESCOLA SUPERIOR DE } \\
\text { ENFERMAGEM SÃO } \\
\text { JOÃO DE DEUS }\end{array}$ & $\begin{array}{c}\text { Reabilitação Psicossocial do Doente com } \\
\text { Depressão }\end{array}$ & $\begin{array}{l}\text { DOMINGUES } \\
\text { HED. }\end{array}$ & $\begin{array}{l}\text { Estratégia de } \\
\text { Intervenção/ } \\
\text { Google } \\
\text { Acadêmico }\end{array}$ \\
\hline $\begin{array}{l}\text { UNIVERSIDADE FEDERAL } \\
\text { DE MINAS GERAIS }\end{array}$ & $\begin{array}{l}\text { Proposta de intervenção para qualificar a } \\
\text { atenção a pessoas com transtornos de saúde } \\
\text { mental na área de abrangência da equipe de } \\
\text { Saúde da Família Cruzeiro do Sul }\end{array}$ & $\begin{array}{l}\text { ZARAGOZA LG. } \\
2017\end{array}$ & $\begin{array}{l}\text { Qualitativo/ } \\
\text { Proposta de } \\
\text { Intervenção/ } \\
\text { Google } \\
\text { Acadêmico. }\end{array}$ \\
\hline $\begin{array}{c}\text { Escola Superior de } \\
\text { Enfermagem de Coimbra }\end{array}$ & $\begin{array}{l}\text { PROMOÇÃO DA SAÚDE MENTAL DAS } \\
\text { PROFESSORAS DO ENSINO BÁSICO: } \\
\text { Contributos do Enfermeiro Especialista nos } \\
\text { Cuidados de Saúde Primários }\end{array}$ & $\begin{array}{l}\text { RODRIGUES } \\
\text { VMSF. }\end{array}$ & $\begin{array}{l}\text { Estudo Pré- } \\
\text { Experimental/ } \\
\text { Google } \\
\text { Acadêmico }\end{array}$ \\
\hline $\begin{array}{l}\text { Internacional Biocentric } \\
\text { Foundation } \\
\text { Escola de Biodanza SRT de } \\
\text { Portugal }\end{array}$ & $\begin{array}{l}\text { Dançar a vida em Saúde Mental: A } \\
\text { biodanza enquanto estratégia em } \\
\text { reabilitação Psicossocial }\end{array}$ & SANTOS MLF. & $\begin{array}{l}\text { Qualitativo/ } \\
\text { Intervenção } \\
\text { Terapêutica/ } \\
\text { Google } \\
\text { Acadêmico }\end{array}$ \\
\hline
\end{tabular}

Fonte: Autor da Pesquisa. 
Tabela 2: Estudos selecionados e suas respectivas condutas de enfermagem recomendadas às mulheres que apresentam sintomas depressivos.

\begin{tabular}{cl}
\hline Autores & \multicolumn{1}{c}{ Resultado principal } \\
\hline $\begin{array}{c}\text { ANDERSON } \\
\text { CA, LIESER }\end{array}$ & $\begin{array}{l}\text { Enfermeira durante Pré Natal deve: identificar os sintomas, referenciar a um especialista } \\
\text { de saúde mental, explicar de forma minuciosa os riscos e benefícios do uso medicamento } \\
\text { antidepressivo durante a gravidez e documentar todo processo. }\end{array}$ \\
C. & $\begin{array}{l}\text { Os principais cuidados da enfermagem são: o envolvimento do paciente com o tratamento, } \\
\text { podendo ser medicamentoso ou terapia, acompanhamento dos sintomas e educação do } \\
\text { paciente. }\end{array}$ \\
SMITH M. \\
BECKIE & $\begin{array}{l}\text { Intervenções individualizadas dedicadas a amparar o comportamento de saúde das } \\
\text { mulheres, são virtualmente mais eficazes. }\end{array}$ \\
TM,et al. \\
SONÇALE & $\begin{array}{l}\text { A ações que os enfermeiros mais relataram foram o acolhimento, 45 (100\%), escuta, 38 } \\
\text { (84,4\%); o orientações, 28 (62,2\%). }\end{array}$ \\
FONTES et al & $\begin{array}{l}\text { Ações de ajuda integral a adolescente grávida, na tentativa de evitar a depressão pós-parto, } \\
\text { através do apego do sujeito deste cuidar com o Recém Nascido. }\end{array}$ \\
CARDOSO & $\begin{array}{l}\text { Em relação ao uso da musicoterapia, dos 48 enfermeiros, 23 revelaram já ter utilizado, } \\
\text { sendo 52, 1\% nunca ter utilizado. Conclui-se que a música é mais utilizada em quadros de } \\
\text { ansiedade, nervosismo, inquietação, insônia, relação diminuída, vontade de viver } \\
\text { diminuída, tendo sido notada melhora em todos os sintomas supracitados nessa ordem. }\end{array}$ \\
AJS. &
\end{tabular}

MENDES Estabeleceu-se um compromisso terapêutico e iniciou-se a intervenção baseada numa AMOC; abordagem comportamental racional emotiva. Estas intervenções possibilitaram a EUFRÁSIO revelação de temores e sobressaltos dos participantes, assim como, a aprendizagem de MLP. como controlar melhor o seu corpo e os seus pensamentos, as suas expectativas e as suas atitudes.

Observou-se que enfermeiros, em atividade na rede básica de saúde (atenção primária), não estão preparados para dar a devida atenção ao portador de transtorno mental, apesar

REIS LA; et de apresentarem médio conhecimento teórico sobre a doença. A adoção de uma prática al que não exclua ações promotoras da saúde mental e atendimento aos casos psiquiátricos precisa estar fundamentado na formação básica do enfermeiro

\begin{tabular}{|c|c|}
\hline $\begin{array}{c}\text { Elias } \\
\text { MTMNC }\end{array}$ & $\begin{array}{l}\text { Foram desenvolvidas competências no âmbito da psicoeducação, tanto ao doente como à } \\
\text { família, de forma ajustada às limitações inerentes à sua situação clínica. Na avaliação das } \\
\text { sessões de relaxamento conseguimos verificar uma relação positiva entre a implementação } \\
\text { dessas técnicas e o alívio da ansiedade, pela avaliação com base nos indicadores (tensão } \\
\text { arterial, respiração, postura), e sustentada pela verbalização de sentimentos positivos pelos } \\
\text { utentes }\end{array}$ \\
\hline $\begin{array}{l}\text { DE } \\
\text { CARVALHO } \\
\text { DRS. }\end{array}$ & $\begin{array}{l}\text { A intervenção realizada terá contribuído nesta doente para diminuir o nível de depressão } \\
\text { e aumentar os conhecimentos sobre esta doença. Vários autores também concluíram que } \\
\text { intervenções psicoeducativas contribuíam para a melhoria dos sintomas depressivos. }\end{array}$ \\
\hline $\begin{array}{l}\text { DOMINGUE } \\
\text { S HED }\end{array}$ & $\begin{array}{l}\text { Os resultados permitem verificar uma melhoria do estado de saúde da doente e da sua } \\
\text { qualidade de vida que se traduz ao nível da diminuição da agitação, da garantia de apoio } \\
\text { social, da diminuição da ansiedade, de uma autoestima mais elevada que no ponto de } \\
\text { partida. Os resultados descendentes (de um score } 53 \text { para um score 19) resultantes da } \\
\text { aplicação do IDB validam os resultados obtidos através do uso de indicadores da NOC, } \\
\text { ou seja, ocorreu uma melhoria significativa do estado de saúde e da qualidade de vida da } \\
\text { doente ao longo do internamento. }\end{array}$ \\
\hline $\begin{array}{c}\text { RODRIGUES } \\
\text { VMSF. }\end{array}$ & $\begin{array}{l}\text { Evolução positiva das dimensões 'Relações Positivas com os Outros' }(p=0,017) \text { e } \\
\text { 'Objetivos de Vida' }(p=0,039) \text { inerentes ao Bem-estar Psicológico, apesar de não se validar } \\
\text { a hipótese formulada na sua globalidade }(p=0,115) \text {; a diminuição da Percepção do Stresse } \\
\text { das professoras }(p=0,000) \text { e diminuição dos níveis de Ansiedade }(p=0,038) \text {. }\end{array}$ \\
\hline $\begin{array}{l}\text { SANTOS } \\
\text { MLF }\end{array}$ & $\begin{array}{l}\text { Pelos resultados obtidos, parece que a Biodanza é uma estratégia com efeitos positivos em } \\
\text { Reabilitação Psicossocial, favorecendo, entre outros, domínios relacionados a Saúde } \\
\text { Física, ao sentimento de prazer e bem-estar, à autoestima, habilidades de interação social }\end{array}$ \\
\hline
\end{tabular}


Fonte: Autor da Pesquisa.

Alguns estudos não participaram da pesquisa por se tratarem de resumos de teses de mestrado, não contendo os resultados procurados; outros por serem projetos de intervenção, não havendo ainda seus resultados.

Nessa revisão foi observado que uma grande parte dos estudos evidenciaram os benefícios da boa relação entre o enfermeiro e paciente, na identificação precoce dos sintomas depressivos e no processo de tomada de decisão para que juntos possam escolher a melhor conduta. Um estudo feito por De Almeida (2014) em idosos com depressão, demonstrou que a enfermagem tem uma importante contribuição na identificação de melhores estratégias de enfrentamento e manejo dos sintomas depressivos, na orientação e incentivo da terapia medicamentosa, o que aumenta a adesão ao tratamento e ajuda na recuperação destes pacientes.

Sendo o profissional que tem uma relação mais restrita com o paciente, o enfermeiro é privilegiado dentro dos grupos de saúde (SILVA et al, 2003). A assistência de enfermagem não se limita em ajudar o paciente, mas também orientar a família e a comunidade (OPAS/OMS, 1997).

Outra conduta recomendada pelos estudos analisados é o tratamento centrado na individualização do sujeito, onde o enfermeiro, através de escuta qualificada, identifica os fatores biopsicossociais que desencadearam os sintomas depressivos para que se aplique uma intervenção específica no problema causador destes sintomas; Duailibi (2014) ressalta a importância do tratamento individualizado na melhora do paciente com depressão.

Segundo um estudo realizado por Silva et al (2003), a maioria dos profissionais que foram entrevistados no estudo $(72,6 \%)$ concordaram que há uma melhora em relação à adesão ao tratamento medicamentoso pelos pacientes quando há uma contribuição da enfermagem.

Essa revisão identificou também que uma das condutas adotadas nos estudos foi à terapia cognitiva comportamental, conduta, esta, que tem uma eficiência comprovada, embora não exclui o tratamento medicamentoso, sempre sendo recomendada a junção desses tratamentos (CARNEIRO et al,2016).

Outra terapia preconizada foi a terapia com música, usada como solução terapêutica para melhorar a saúde mental, sedo uma terapia alternativa dinâmica e de baixo custo (CÂMARA e CAMPOS, 2013).

A dança foi citada em um estudo como uma prática favorável no tratamento da depressão. O autor Alencar (2006) cita a biodança como uma forma de integração de pessoas, 
que por consequência melhora a vida, dando mais qualidade a essas pessoas, independentemente de sua classe social.

Foi encontrado um artigo, no qual incentiva o enfermeiro referenciar a paciente que apresente sintomatologia depressiva a um especialista em saúde mental, pois segundo Souza et al (2007), uma grande parte dos enfermeiros não têm especialidade em saúde mental, em consequência, esses profissionais, os quais são generalistas, sentem maiores dificuldades no planejamento do atendimento dessas pessoas.

O que facilitou esse encaminhamento e referência foi a criação do CAPS (Centro de Atenção Psicossocial) que, segundo Brasil (2011), é um ambiente aberto ao público onde é composto por uma equipe multiprofissional à serviço da comunidade e de pessoas com transtornos mentais. Com isso, ao serem detectadas nessas mulheres sintomas depressivos, os profissionais podem encaminhar para esses serviços.

\section{Conclusão}

A área da saúde é um setor em constante mudança, sendo necessário uma renovação contínua também das evidências científicas. Percebe-se que há uma enorme necessidade de mais estudos relacionados à assistência de enfermagem frente a mulheres com sintomas depressivos.

É preciso melhorar a assistência e conduta da enfermagem para essa problemática, pois foram poucos os estudos que demonstraram os resultados das intervenções realizadas. Deve-se salientar que uma boa assistência de enfermagem é de suma importância para detecção precoce da sintomatologia depressiva, seguida por intervenções imediatas para os casos mais graves. Essa assertiva contribui para melhores resultados do tratamento, pois entende-se que os resultados dos estudos analisados mostraram a importância do profissional da enfermagem no cuidado e acolhimento de mulheres com sintomatologia depressiva.

\section{Referências}

ALENCAR, Bárbara P.; MENDES, Maria M. R.; JORGE, Maria S. B.; RODRIGUES, Maria S. P. Significado da Biodança como fonte de Liberdade e Autonomia na auto-reconquista 
no Viver Humano. Revista Texto \& Contexto Enfermagem. Florianópolis, p 48 - 54, n. 15, 2006.

American PsychiatryAssociation. DiagnosticandStatistical Manual of Mental disorders DSM-5. 5th.ed. Washington: American PsychiatricAssociation, 2013.

American PsychologicalAssociation. Policystatementonevidence-basedpractice in psychology. American PsychologicalAssociation; 2005.

ANDERSON CA; LIESER C. Prenatal depression: Early intervention. The Nurse Practitioner. Vol. 40, No. 7. 2015.

Associação Brasileira de Psiquiatria - ABP. OMS: depressão será a doença mais comum do mundo em 2030 [Internet]. Teresina PI, 2014. Disponível em:

http://www.capitalteresina.com.br/noticias/saude/oms-depressao-sera-a-doenca-mais-comumdo-mundo-em-2030-21064.html

Beckie, T. M.; Beckstead, J. W.; Schocken, D. D.; Evans, M. E.; Fletcher G. F. The effects of a tailored cardiac rehabilitation program on depressive symptoms in women: A randomized clinical trial. International Journal of Nursing Studies 48 (2011) 3-12. 2011.

CÂMARA, Yzy Maria Rabelo; CAMPOS, Maria dos Remédios Moura. Musicoterapia como recurso terapêutico para a saúde mental. Cadernos Brasileiros de Saúde Mental/BrazilianJournalof Mental Health, v. 5, n. 12, p. 94-117, 2013.

CARDOSO AJS. A utilização da música como coadjuvante terapêutico na Saúde Mental e Psiquiatria. Universidade Fernando Pessoa; Faculdade de Ciências da Saúde. 2010

DE ALMEIDA, Mariana Figueiredo Inez et al. Depressão do idoso: o papel da assistência de enfermagem na recuperação dos pacientes depressivos. Revista Eletrônica Interdisciplinar, v. 1, n. 11, 2014.

DE CARVALHO DRS. Redescobrindo a Enfermagem de Saúde Mental. Instituto Politécnico de Leiria- Escola Superior de Saúde. 2015.

Dias MMG. Intervenção em Enfermagem de saúde mental e Psiquiátrica num centro de dia do concelho de Oeiras. Universidade Católica Portuguesa Instituto de Ciências da Saúde. 2011.

DOMINGUES HED. Reabilitação Psicossocial do Doente com Depressão. UNIVERSIDADE DE ÉVORA ESCOLA SUPERIOR DE ENFERMAGEM SÃO JOÃO DE DEUS. 2016.

DUAILIBI, Kalil; DA SILVA, Anderson Sousa Martins. Depressão: critérios do DSM-5 e tratamento. Rev. Bras. Clin. Terap, v. 40, n. 1, p. 27-32, 2014.

FONSECA, J. J. S. Metodologia da pesquisa científica. Fortaleza: UEC, 2002. Apostila Elias MTMNC. As Técnicas de Relaxamento como Instrumento Terapêutico de Enfermagem em Pessoas com Sofrimento Mental. Escola Superior de Enfermagem de Lisboa. 2014. 
Fontes, F. S.; Silva, I. A.; Ribeiro, I. B.; Lima, G. P. V.; Guida, N. F. B. O cuidado de enfermagem e sua contribuição para prevenir a depressão pós-parto na adolescência. Revista de Pesquisa: Cuidado é Fundamental Online. 2010

GONÇALVES, RMDA. Ações dos Enfermeiros em Saúde Mental na Estratégia Saúde da Família. Dados Internacionais de Catalogação-na-Publicação (CIP) (Biblioteca Frei Eugênio, Universidade Federal do Triângulo Mineiro, MG, Brasil). 2009

GUEDES, C.R; ALVARENGA, B.D.D; ROTELLA, I; VILELLA, D.V.A.L. Habilidades do Enfermeiro no Diagnóstico e Cuidado ao Portador de Depressão. Revista Ciências em Saúde v5, n 4, 2015. Minas Gerais, Brasil

HOFMANN SG, ANU ASNAANI MA, HINTON DE. Cultural aspects in social anxietyand social anxietydisorder.DepressAnxiety 2010; 27(12): 1117-27.

MENDES AMOC; EUFRÁSIO MLP. Análise compreensiva de uma intervenção na ansiedade e depressão em doentes hospitalizados com insuficiência cardíaca. Revista de Enfermagem Referência. 2013.

Métodos de pesquisa / [organizado por] Tatiana Engel GERHARDT e Denise Tolfo SILVEIRA; coordenado pela Universidade Aberta do Brasil - UAB/UFRGS e pelo Curso de Graduação Tecnológica - Planejamento e Gestão para o Desenvolvimento Rural da SEAD/UFRGS. - Porto Alegre: Editora da UFRGS, 2009.

Ministério da Saúde. Portaria GM/MS n 3.088, de 23 de dezembro de 2011. Republicada em 21 de maio de 2013. Institui a Rede de Atenção Psicossocial para pessoas com sofrimento ou transtorno mental, incluindo aquelas com necessidades decorrentes do uso de crack, álcool e outras drogas, no âmbito do Sistema Único de Saúde (SUS). Brasília, 2011.

OPAS/OMS. Programa de Salud Mental, División de Promócion de Salud. Modelo para lacapacitación de laenfermeria general en al identificación y manejo de los transtornos afectivos. Generalista I; 1997.

ORGANIZAÇÃO MUNDIAL DA SAÚDE. Classificação de transtornos mentais e de comportamento da CID-10: Descrições clínicas e diretrizes diagnósticas. Porto Alegre: Artes Médicas, 1993.

PEREIRA, I. F; FARIA, L. C; VIANNA, R. S. M; CORREAA,P. D. S; FREITAS,D. A; SOARES, W. D. Depressão e uso de medicamentos em profissionais de enfermagem. Arq. Ciênc. Saúde. 2017 jan-mar; 24(1) 70-74

REIS LA; BRITO FR; MOREIRA VS; AGUIAR ACSA. Atuação do Enfermeiro do Programa de saúde da Família frente ao indivíduo portador de transtorno mental. C\&DRevista Eletrônica da Fainor, Vitória da Conquista, v.6, n.2, p.175-187, jul./dez. 2013.

RODRIGUES VMSF. PROMOÇÃO DA SAÚdE MENTAL DAS PROFESSORAS DO ENSINO BÁSICO: Contributos do Enfermeiro Especialista nos Cuidados de Saúde Primários. Escola Superior de Enfermagem de Coimbra. 2017. 
Santiago A, Holanda AF. Fenomenologia da depressão: uma análise da produção acadêmica brasileira.RevAbordagemGestált. 2013;19(1):38-50.

SANTOS MLF. Dançar a vida em Saúde Mental: A biodanza enquanto estratégia em reabilitação Psicossocial. Internacional Biocentric Foundation Escola de Biodanza SRT de Portugal. 2017.

SEQUEIRA, C. Editorial: Consensos em Saúde Mental - Diagnósticos, Intervenções e Resultados. 2014.

Silva MCF, Furegato ARF, Costa ML Júnior. Depressão: pontos de vista e conhecimento de enfermeiros da rede básica de saúde.Rev Latino-am Enfermagem 2003 janeiro-fevereiro; 11(1):7-13.

SMITH M. Collaborative Care Models for Late-Life Depression and Anxiety. Journal of Gerontological Nursing. Vol. 36, No. 9, 2010. 2010

SOUTHWICK SM, CHARNEY DS. The Science ofresilience: implications for thepreventionandtreatmentofdepression.Science 2012; 338(6103): 79-82

Souza AJF, Matias GN, Gomes KFA, Parente ACM. A saúde mental no Programa de Saúde da Família. Ver Bras. Enfermagem 2007 julho-agosto; 60(4):391-5.

Waidman MAP. Marcon SS, Pandini A, Bessa JB, Paiano M. Assistência de enfermagem às pessoas com transtornos mentais e às famílias na atenção básica.Acta Paul Enferm. 2012;25(3):346-51.

ZAMMIT S, OWEN MJ. Stressfullifeevents, 5-HTT genotypeandriskof depression. $\mathrm{Br}$ J Psychiatry 2006; 188: 199-201

ZARAGOZA LG. Proposta de intervenção para qualificar a atenção a pessoas com transtornos de saúde mental na área de abrangência da equipe de Saúde da Família Cruzeiro do Sul. UNIVERSIDADE FEDERAL DE MINAS GERAIS. 2017.

Como citar este artigo (Formato ABNT):

GOMES, Thainá Emí B.; IVO, Olguimar P. Sistematização da Assistência de Enfermagem em Mulheres com Sintomatologia Depressiva: Uma Revisão Sistemática. Id on Line Revista Multidisciplinar e de Psicologia, 2017, vol.11, n.38, p. 835-848. ISSN: 1981-1179.

Recebido: 08.11.2017

Aceito: 11.11.2017 